\title{
Comprehensive Clinical Assessment in Community Setting: Applicability of the MDS-HC
}

\author{
John N. Morris, PhD, Brant E. Fries, PhD, ${ }^{\dagger}$ Knight Steel, $M D,{ }^{\dagger}$ Naoki Ikegami, MD, \\ Roberto Bernabei, $M D, " G$. Iain Carpenter, $M D, "$ Ruedi Gilgen, MD," \\ John P. Hirdes, PhD, ${ }^{+\dagger}$ and Eva Topinková, $M D^{\neq \neq}$
}

\begin{abstract}
OBJECTIVE: To describe the results of an international trial of the home care version of the MDS assessment and problem identification system (the MDS-HC), including reliability estimates, a comparison of MDS-HC reliabilities with reliabilities of the same items in the MDS 2.0 nursing home assessment instrument, and an examination of the types of problems found in home care clients using the MDS-HC.
\end{abstract}

DESIGN: Independent, dual assessment of clients of homecare agencies by trained clinicians using a draft of the MDS$\mathrm{HC}$, with additional descriptive data regarding problem profiles for home care clients.

SETTING AND PARTICIPANTS: Reliability data from dual assessments of 241 randomly selected clients of home care agencies in five countries, all of whom volunteered to test the MDS-HC. Also included are an expanded sample of 780 home care assessments from these countries and 187 dually assessed residents from 21 nursing homes in the United States.

MEASUREMENTS: The array of MDS-HC assessment items included measures in the following areas: personal items, cognitive patterns, communication/hearing, vision,

\footnotetext{
From the "Hebrew Rehabilitation Center for Aged, HRCA Research and Training Institute, Boston, Massachusetts; tDepartment of Gerontology and School of Public Health, University of Michigan and VA Medical Center, Ann Arbor, Michigan; $¥$ New Jerscy Medical School, Hackensack University Medical Center, Hackensack, New Jersey; SDepartment of Health Policy and Management, Keio University School of Medicine, Tokyo, Japan; UUniversità Cattolica del Sacro Cuore, Facoltà di Medicina e Chirurgia "Agostino Gemelli", Rome, Italy; |Center for Health Service Studies, The University of Kent, Canterbury, Great Britain; \#Klinik fur Geriatrie und Rehabilitation, Zurich, Switzerland; ††Department of Health Studies and Gerontology, University of Waterloo, Ontario, and Canadian Collaborating Centre --inter RAI Providence Centre, Scarborough, Ontario; and $\ddagger$ Department of Geriatric Medicine, Postgraduate School of Medicine, Czech Republic.

Drs. Morris' and Fries' work on this paper was supported, in part, by an Alzheimer's Association Award \#TRG-93-022, "Assessment and Outcomes for Community-Based Cognitively Impaired Elderly." Dr. Morris holds the Alfred A. and Gilda Slifka Chair in Social Gerontological Research. Dr. Steel's work was supported in part by a grant from the John A. Hartford Foundation, Inc., "Expansion of Homecare into Academic Medicine," and the Hunterdon Health Fund. Dr. Ikegami's work was supported, in part, by a grant from the Ministry of Health and Welfare of Japan, "Improving the Health of the Flderly" project, allocated by the Health Insurance Association.

Address correspondence to John N. Morris, PhD, Hebrew Rehabilitation Center for Aged, HRCA Research and Training Institute, 1200 Centre St, Boston, MA 02131 .
}

mood and behavior, social functioning, informal support services, physical functioning, continence, disease diagnoses, health conditions and preventive health measures, nutrition/ hydration, dental status, skin condition, environmental assessment, service utilization, and medications.

RESULTS: Forty-seven percent of the functional, health status, social environment, and service items in the MDS-HC were taken from the MDS 2.0 for nursing homes. For this item set, it is estimated that the average weighted Kappa is .74 for the MDS-HC and .75 for the MDS 2.0. Similarly, high reliability values were found for items newly introduced in the MDS-HC (weighted Kappa $=.70$ ). Descriptive findings also characterize the problems of home care clients, with subanalyses within cognitive performance levels.

CONCLUSION: Findings indicate that the core set of items in the MDS 2.0 work equally well in community and nursing home settings. New items are highly reliable. In tandem, these instruments can be used within the international community, assisting and planning care for older adults within a broad spectrum of service sertings, including nursing homes and home care programs. With this community-based, secondgeneration problem and care plan-driven assessment instrument, disability assessment can be performed consistently across the world. J Am Geriatr Soc 45:1017-1024, 1997.
For nursing home residents, a first step in the design of a standardized assessment system can be seen in the introduction of the Resident Assessment Instrument (RAI) in the United States and elsewhere. ${ }^{2-5}$ Major subcomponents of 
that assessment system have been validated and positive patient outcomes documented. ${ }^{6-8}$ For home care, we explored whether there were comparable "second generation" assessment instruments that include both problem identification and care plan specification components. We found that community-based instruments tend to be less comprehensive. For example, in a review of 50 community-based assessment instruments in the UK, conceptual omissions were common, standardized response sets were rarely used, and only $24 \%$ of the instruments were employed by both social and health care workers. ${ }^{9}$

Although no single comprehensive assessment battery has been created, within medical geriatrics, geriatricians have reported extensively on the efficacy of geriatric assessment programs. ${ }^{10,11}$ The results have varied, ${ }^{12-14}$ with some evidence from the international community that comprehensive assessments can be used to reduce the risk of mortality, acute hospitalization, and other adverse health outcomes. ${ }^{15}$ At the same time, ". . . the literature. . . [identifies] very few instruments with demonstrated validity and reliability among very old community persons." ${ }^{11}$ Within this environment, as geriatricians have been compelled to create ad hoc instruments, there has been a clear preference for multidimensional assessment systems that incorporate functional, social, disease, and environmental measures ${ }^{16-20}$; however, there has also been considerable discontinuity in the recommended item sets. ${ }^{21}$

\section{Description of the RAI-HC}

The RAI-HC, as ultimately constructed, consists of two elements:

- The MDS-HC assessment component enables the home care clinician to assess multiple domains of function, health, social support, and service use. In addition, selected subsets of MDS-HC items provide a standardized mechanism to identify persons who could benefit from further evaluation of specific problems or risk for functional decline; these items are called triggers.

- The Clinical Assessment Protocols (CAPs) provide general guidelines for further assessment and individualized care planning of triggered problems. They include background (with prevalence data, etc.) and care planning guidelines to serve as a training manual and reference for the home care professional.

Work on the MDS-HC was initiated in January 1993 by an international group of clinicians and researchers who had collaborated to promote research on the RAI for nursing homes (inter $R A I$ ). The interRAI group believed that the populations served by nursing homes and home care programs overlap, that many of the RAI assessment items should be applicable in the community, and, ultimately, it set design parameters for the RAI-HC that require that it: ${ }^{22}$ (1) Be client based, with a built in capacity for following clients over time; (2) Support care planning; (3) Be usable across multiple nations; (4) Provide the basis for future development of outcome measures; (5) Serve as a training manual and reference for home care professionals; (6) Support a broad, multidisciplinary standard of care; and, (7) Be capable of being completed within 1 hour (the assessment and recoding of information).

The logic of construction of the RAI-HC mimics the problem-based approach used in the RAI for nursing homes. For the nursing home RAI, Resident Assessment Protocols
(RAPs) were created to support problem identification and care planning. Similarly, for the home care RAI-HC system, the 30 CAPs were created to cover a broad range of functional, clinical, and environmental problems.

The initial item configuration of the MDS-HC consisted of the CAP trigger elements, with additional items included to cover key demographic conditions, disease status, and service utilization. In addition, working with an advisory committee of the National Association for Home Care in the United States, items were added to allow the instrument to relate better to the post-hospital, discharge-driven environment.

\section{Field Operational Guidelines for Use of RAI-HC}

The RAI-HC is designed for use by clinical professionals: nurses, social workers, therapists, and physicians. It is not a questionnaire, although its use does require direct questioning of the older person and his or her primary informal caregiver (if available). The assessor also observes the individual in the home environment and reviews other healthrelated documents, when available. The items on the MDS-HC flow in what is presumed to be a reasonable sequence although the assessor is not bound by their order. When answers appear to be of questionable validity, the assessor may carry out a further examination of relevant areas. It is the composite of this information that is to be recorded on the MDS-HC. Finally, it is possible to break the assessment process into two parts, gathering the required information on two visits over a 7 to 14 -day period.

\section{Mapping MDS and MDS-HC Item Sets}

Table 1 charts the item comparability between the MDS-HC and MDS 2.0 used in nursing homes. Of the 223 functional, health status, social environment and service items in the MDS-HC, 114 (47\%) come from the current Version 2.0 of the MDS for nursing homes. In the key areas of cognition, communication, vision, mood, behavior, ADL self-performance and continence, 30 of the $32 \mathrm{MDS}-\mathrm{HC}$ items come from MDS 2.0.

Newly created items for the MDS-HC are largely representative of areas that are encountered less frequently in the nursing home setting. These include the role of informal supports, indicators of abuse of the older person, IADL self-performance, environmental conditions, a variety of health conditions including preventative health measures and alcohol abuse, and a series of service use indicators.

In this article, we present the outcomes of a crossnational field trial with data from Australia, Canada, the Czech Republic, Japan, and the United States. Two major issues are addressed: (1) the reliability of the MDS-HC item set, including a comparison with the reliability of the items that are common to MDS 2.0 in use in nursing homes; and (2) an examination of the complexity of the problem configuration observed for home care clients using the MDS-HC. In this latter presentation, we describe the CAP problem profiles for persons cared for in home care programs in our crossnational home care sample.

\section{METHODS}

\section{Reliability Trial}

The home care reliability trial involved dual, independent assessments of older home care clients in five countries: Australia, Canada, the Czech Republic, Japan, and the 
Table 1. Comparability of Substantive (Nondemographic) Items on the MDS-HC and the MDS 2.0 for Nursing Homes

\begin{tabular}{lcc}
\hline \multicolumn{1}{c}{ MDS-HC Domain } & $\begin{array}{c}\text { Total Number of Items in } \\
\text { Domain for MDS-HC }\end{array}$ & $\begin{array}{c}\text { Total Number of Overlapping Items from } \\
\text { MDS for Nursing Homes }\end{array}$ \\
\hline B. Cognitive patterns & 4 & 3 \\
C. Communicating/Hearing patterns & 3 & 3 \\
D. Vision patterns & 3 & 2 \\
E. Mood and behavior patterns & 12 & 11 \\
F. Social functioning & 5 & 2 \\
G. Informal support services & 15 & 0 \\
H. Physical functioning & 7 & \\
IADL self performance & 8 & 0 \\
ADL self performance & 5 & 8 \\
$\quad$ Locomotion/Stamina & 3 & 0 \\
Functional potential & 4 & 2 \\
I. Continence & 27 & 4 \\
J. Disease diagnosis & 39 & 23 \\
K. Health conditions & 8 & 12 \\
L. Nutrition/Hydration status & 3 & 5 \\
M. Dental status & 21 & 1 \\
N. Skin condition & 10 & 12 \\
O. Environmental assessment & 52 & 0 \\
P. Service utilization & 9 & 5 \\
Q. Medications & 238 & 19 \\
Total items & & 112 (47.1\%) \\
\hline
\end{tabular}

United States. Samples were drawn from agencies willing to participate in the trial and thus do not constitute a random sample of all older people served in these countries. The resulting home care reliability sample is composed of 241 dual assessments, $47 \%$ from Japan, $28 \%$ from the United States, $11 \%$ from Canada, $10 \%$ from Australia, and $4 \%$ from the Czech Republic. In each agency, two assessors (usually nurses, but including social workers, therapists, and physicians) completed assessments of a randomly selected sample of individuals independently, with the reassessments scheduled to occur within a 7-day period. Assessors prepared for their role in testing the MDS-HC by reviewing the User's Manual that described the instrument. They next completed a preliminary practice assessment following the specified data acquisition and coding processes described in the User's Manual. They were then debriefed to ensure that they were prepared to complete the MDS-HC assessments in accordance with the item specifications. The two assessors at each agency next completed independent assessments of a random sample of clients served by the agency. Ten older adults were scheduled at each agency. In completing these cases, the two assessors did not discuss the older people or their findings. The data that emerged from this field test permitted a comprehensive evaluation of the reliability of the MDS-HC items. In the reliability analyses, weighted Kappa values are presented. To put these values into perspective, Kappa values lower than 0.4 indicate poor reliability, values between .40 and .75 are considered adequate, and values of .75 or above are considered evidence of excellent reliability. ${ }^{2.3}$

In addition, for comparative purposes, for items in the MDS-HC that were drawn from the MDS 2.0 for nursing homes, we compared the reliability of these items across the two settings. The nursing home reliability cases were drawn from a 21-site field test in the United States. ${ }^{5}$ A total of 187 dual assessments were used, with each resident being assessed independently by two nurse assessors, each of whom had previous MDS experience. There was a total of 42 nurse assessors.

\section{Complexity Analysis}

One additional sample was used in our analysis of the complexity of problem configurations. More specifically, the MDS-HC sample was supplemented by other cases from Canada, Japan, and the United States, resulting in an ultimate sample of 780 cases, including the 241 cases used in the reliability trial (for the latter cases, we used the assessment of one of the two clinical assessors for each of the dually reviewed reliability cases).

The complexity of the problem configurations was based on an examination of the frequency with which CAPs were triggered in the 780-case home care sample in the presence and absence of cognitive impairment. For these analyses, the cognitive index used is the Cognitive Performance Scale (CPS) ${ }^{24}$ which is based on a subset of items included in the MDS-HC. The CPS scale has seven levels, representing persons who are cognitively Intact (CPS levels of 0,1 ), those who have Mild to Moderately Impairment (CPS levels of 2,3) and those who are Severely Impaired (CPS levels of 4-6).

\section{RESULTS}

\section{Sample Description}

The mean age of the full home care client sample is 79.6 years; $59.5 \%$ are female, $37.9 \%$ are married, $26.0 \%$ did not go out of the house in the week before the assessment, and $32.1 \%$ live alone. Using the categories of the Cognitive Per- 
formance Scale, $41.2 \%$ are Intact (representing persons who have been shown in earlier work to have average Folstein Mini-Mental Examination scores of $>25$, where 30 is the best performance), whereas at the other extreme of the 7-level CPS, $7.4 \%$ are Severely Impaired and $3.6 \%$ are Very Severely Impaired (the latter representing a category that earlier studies have shown to have an effective Mini-Mental Examination score close to zero). A total of $61.7 \%$ have multiple health diagnoses, including $25.4 \%$ with stroke, $7.6 \%$ hip fracture, and $11.4 \%$ cancer. At the time of assessment, $23.7 \%$ were receiving a therapeutic service (exercise, physical, occupational, or speech therapy), and $31.7 \%$ were receiving one or more special treatments (e.g., chemotherapy, dialysis, oxygen supplementals, skin care, or the use of a respirator).

The nursing home reliability comparison sample is older (mean age 82.5 years), more are female $(75.4 \%$ ), and more are Severely Impaired on the Cognitive Performance Scale
$(17.2 \%) ; 98.2 \%$ have at least one health diagnosis, $30.1 \%$ have five or more diagnoses, and of the specific diagnoses, $24.7 \%$ have a stroke, $18.5 \%$ congestive heart failure, and $6.5 \%$ Parkinson's Disease. Finally, $12.6 \%$ were receiving a therapeutic service (about one-half the rate in the home care sample), and $63 \%$ were receiving one or more special treatments (skin care being the most common).

\section{Reliability Results}

Table 2 compares reliability results of the MDS-HC (for home care) and the MDS 2.0 (for nursing homes) for 93 of the 114 common items. (Note: reliabilities are unavailable for the remaining 21 items either because of a lack of variation among subjects or in some cases, because of an absence of the item from the earlier version of the MDS-HC on which these reliability cases were gathered). In general, the MDS-HC reliabilities are comparable to those found for residents assessed in nursing homes using MDS 2.0.5 The average

Table 2. Reliability Cross Walk of the Identical Items MDS-HC and MDS 2.0 (Weighted Kappas for Areas in Common)

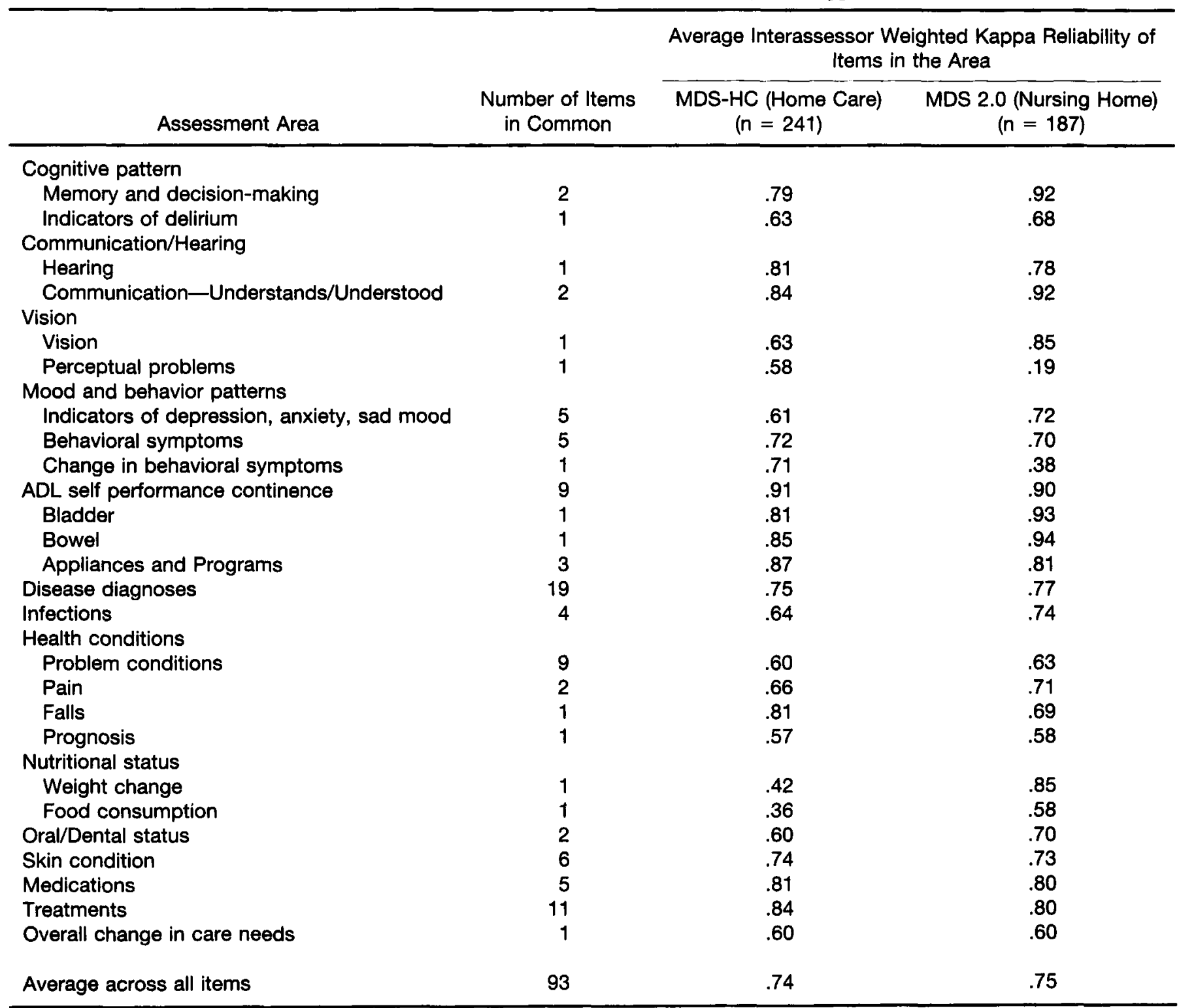


weighted Kappa across the item set is .74 for the MDS-HC and .75 for the MDS 2.0. An ability to attain reliable answers to these items is thus not specific to the nursing home setting.

Table 3 summarizes the weighted Kappa values for items newly introduced in the MDS-HC. For the 57 items in Table 3 , the average weighted $\mathrm{Kappa}$ is . 70 , ranging between .49 for the two danger of falls measures to .79 for the four indicators of elder abuse measures. Pooling the reliability values from Tables 2 and 3, the average MDS-HC item had a reliability of .72 , which can be compared with an average of .79 for the nursing home MDS 2.0 instrument. $^{5}$

\section{Complexity of Problem Configuration Identified by the MDS-HC.}

Table 4 presents information on the configuration of problem conditions identified on the basis of the 30 CAP triggers. Estimates are provided for the total sample (which is, of course, not an international, representative sample), and for persons within the categories of the Cognitive Performance Scale (representing distinct types of persons, and, thus, less subject to inter-sample fluctuations). For the average home care client in the 780-person sample, 11.8 CAPs were triggered. For this sample, only $5.5 \%$ triggered five or fewer CAPs, whereas $2.1 \%$ triggered 20 or more CAP areas. The most prevalent conditions triggered include preventative health measures $(87 \%)$, IADL rehabilitation $(83 \%)$, falls $(79 \%)$, social function $(77 \%)$, and health promotion $(74 \%)$. The least prevalent conditions triggered were abuse of older adults $(7.9 \%)$, adherence to treatments or procedures $(5.8 \%)$, palliative care, $(4.1 \%)$ and alcohol abuse $(2.9 \%)$. These profiles are significantly different for clients at either extreme of the cognitive performance continuum: the more intact clients trigger an average of $10.8 \mathrm{CAPs}$ areas; the most severely impaired trigger an average of 13.6 CAPs. In this comparison, two factors stand out: clients with severe cognitive deficits trigger more frequently in areas such as institutional risk, communication disorders, cognitive problems, pressure ulcers, bowel management, and urinary inconti- nence. CAP areas where the cognitively intact clients are more likely to trigger include IADL rehabilitation, social function, cardiorespiratory, falls, and pain.

\section{DISCUSSION}

This paper describes the MDS for home care, an internationally developed, second-generation, problem and care plan guideline-driven assessment instrument for use in community care settings. The instrument is designed to assist clinicians in arriving at a comprehensive view of the needs and strengths of the population served, a view that is essential to the development of appropriate plans of care allowing older adults to achieve their functional potential. ${ }^{3,25}$ The MDS-HC is an extension of the MDS Version 2.0 for use in nursing homes and, together these instruments can work in tandem to track older people within vertically integrated healthcare systems. This system thus forms the basis for a common core of key assessment items for following older persons from institutional to community settings.

The findings on the reliability of the MDS-HC, both in its own right and in comparison with the MDS 2.0 used in nursing homes, are significant as one contemplates the future use of this instrument around the world and the need to assess carefully the degree of commonality in the problem and disability distribution of older people in different countries. In this study, the internationally developed and tested MDS-HC begins by incorporating a sub-set of MDS $2.0 \mathrm{items}$ whose reliability levels have now been shown to be comparable to those reported previously for the items when applied to nursing home residents. We have demonstrated that these items are equally applicable when used with clients of home care agencies and residents in nursing homes. This system for assessment is thus not a limited site-specific tool. Rather, these findings demonstrate that the assessment tool is personspecific, with many core items proven applicable in both community and nursing home settings. With this communitybased system, disability assessment can be performed consistently around the world, and by using the logic of the CAP

Table 3. Weighted Kappa Reliabilities for Items Newly Introduced in the MDS-HC

New Assessment Areas in the MDS-HC That Are Not in the MDS 2.0
Number of Items in Weighted Kappa Assessment
Average Interassessor Reliability of Items in the Domain
F. Social functioning

G. Informal support services

4

IADL self performance

IADL difficulty (capacity)

Other (stair climbing, stamina)

K. Health conditions and preventive health measures

Preventive health

Problem conditions

Danger of falls

Health status indicators

Other status indicators (indicators of elder abuse)

M. Dental status

O. Environmental assessments

P. Service utilization
5

7

7

2

4

4

2

2

4

2

6

8

\section{.68}

.66

.77

.76

.74

.52

.67

.49

.58

.79

.57

.71

.75 
Table 4. Percent Triggered on Clinical Assessment Protocols (CAPs) Within Categories of "Cognitive Performance Scale"

\begin{tabular}{|c|c|c|c|c|c|}
\hline & \multicolumn{5}{|c|}{ Cognitive Performance Scale (CPS) } \\
\hline & $\begin{array}{c}\text { Total } \\
\text { Sample } \\
N=780\end{array}$ & $\begin{array}{c}\text { Intact } \\
0,1 \\
n=451\end{array}$ & $\begin{array}{c}\text { Mild to } \\
\text { Moderate } \\
(2,3) n=190\end{array}$ & $\begin{array}{c}\text { Severe } \\
(4-6) \\
n=117\end{array}$ & $\begin{array}{l}\text { Chi Square } \\
\text { Significance } \\
\text { Across CPS }\end{array}$ \\
\hline \multicolumn{6}{|l|}{ CAPs related to functional performance } \\
\hline ADL/Rehabilitation potential & $31.4 \%$ & $27.3 \%$ & $50.3 \%$ & $21.4 \%$ & .001 \\
\hline Instrumental Activities of Daily-Living (IADLs) & 83.4 & 94.0 & 92.1 & 28.2 & .001 \\
\hline Health promotion & 73.7 & 80.3 & 86.3 & 28.2 & .001 \\
\hline Institutional risk & 54.0 & 38.6 & 64.7 & 95.7 & .001 \\
\hline \multicolumn{6}{|l|}{ CAPs related to sensory performance } \\
\hline Communication disorders & 62.1 & 40.8 & 89.5 & 100.0 & .001 \\
\hline Visual function & 45.0 & 39.9 & 51.6 & 53.8 & .01 \\
\hline \multicolumn{6}{|l|}{ CAPs related to mental health } \\
\hline Alcohol abuse and hazardous drinking & 2.9 & 2.4 & 2.6 & 5.1 & \\
\hline Cognition & 45.4 & 16.9 & 79.5 & 100.0 & .001 \\
\hline Behavior & 11.2 & 3.8 & 17.9 & 29.1 & .001 \\
\hline Depression and anxiety & 14.8 & 10.0 & 18.4 & 27.4 & .001 \\
\hline Elder abuse & 7.9 & 3.5 & 12.6 & 17.1 & .001 \\
\hline Social function & 77.3 & 82.0 & 70.5 & 70.1 & .001 \\
\hline \multicolumn{6}{|l|}{ CAPs related to health problems/syndromes } \\
\hline Cardiorespiratory & 39.6 & 42.6 & 40.5 & 26.5 & .01 \\
\hline Dehydration & 22.0 & 21.7 & 18.9 & 28.2 & \\
\hline Falls & 78.8 & 82.5 & 78.4 & 65.0 & .001 \\
\hline Nutrition & 21.9 & 21.5 & 20.5 & 25.6 & \\
\hline Oral health & 29.6 & 25.9 & 32.1 & 39.3 & .01 \\
\hline Pain & 52.6 & 60.1 & 50.5 & 27.4 & .001 \\
\hline Pressure ulcers & 37.5 & 28.6 & 36.8 & 72.0 & .001 \\
\hline Skin and foot condition & 43.9 & 44.3 & 40.5 & 47.9 & \\
\hline \multicolumn{6}{|l|}{ CAPs related to continence } \\
\hline Bowel management & 36.7 & 27.9 & 32.6 & 76.9 & .001 \\
\hline Urinary incontinence and indwelling catheter & 42.1 & 31.0 & 42.6 & 83.8 & .001 \\
\hline \multicolumn{6}{|l|}{ CAPs related to service oversight } \\
\hline Adherence & 5.8 & 2.9 & 7.4 & 14.5 & .001 \\
\hline Brittle support system & 35.4 & 34.1 & 37.9 & 35.9 & \\
\hline Medication management & 24.8 & 27.5 & 20.5 & 21.4 & \\
\hline Palliative care & 4.1 & 3.3 & 4.2 & 6.8 & \\
\hline Preventive health measures: Immunization and screening & 86.7 & 83.4 & 89.5 & 94.9 & .01 \\
\hline Psychotropic drugs & 16.6 & 15.1 & 22.1 & 13.7 & \\
\hline Reduction of formal services & 55.0 & 54.4 & 60.5 & 59.0 & \\
\hline Environmental assessment & 41.0 & 39.5 & 44.7 & 41.0 & \\
\hline Average CAPs triggered & 11.8 & 10.8 & 13.2 & 13.6 & .001 \\
\hline Standard deviation & 3.6 & 3.3 & 4.0 & 3.1 & \\
\hline
\end{tabular}

triggering process, care planning can be approached uniformly. The trained clinician will follow a common examination protocol in New York, Milan, and Tokyo.

The decision making and communication measures included in the MDS-HC can be used to track the progression of cognitive loss as clients are exposed to care programs. The weighted Kappas for these items all exceed the .75 standard for excellent reliability: Decision making (.93 NH, .87 HC); Making self understood $(.92, .85)$; and Ability to understand others $(.92, .83)$.

Other indicators shown to work with equal utility in both nursing home and home care settings are the various functional measures. Weighted Kappas were universally high. As markers of decline, loss of independence in the home care sample is greater in "early loss" ADLs, $27.9 \%$ are independent in bathing and $49.9 \%$ are independent in dressing, than in "late-loss" ADLs, $72.9 \%$ are independent in bed-mobility and $74.3 \%$ are independent in eating. Total dependency levels for bed mobility occur for $8.7 \%$ in home care $(25.3 \%$ in nursing homes), whereas for eating the rate is $5.4 \%$ in home care $(21.4 \%$ in nursing homes).

The MDS-HC, while linking to its sister instrument for nursing homes, also contains many unique domains that make it suited for use in the community setting. Several of the community-specific elements of the MDS-HC were newly created for the MDS-HC, whereas others are based on previ- 
ously established measures. However, in both instances, the new items achieve reasonable reliability. More specifically, note the inclusion in the MDS-HC's of reliable indicators of stamina (average weighted Kappa $=.74)$, alcohol use (.79), daily smoking (.91), elder abuse (.75), and compliance/ adherence with medication prescriptions (.84).

At the same time, not all of the measures tried in earlier versions of the MDS-HC were successful. More specifically, three measures addressing the issue of sexuality had very low reliability (with an average Kappa of about zero), and are now excluded from the instrument. These questions addressed the older person's problem with sexual patterns (including sexual companionship), refraining from sexual activity because of assumed health and other risks (including body image and privacy problems), and reports of recent decrease in sexual activity. In general, about $3 \%$ of home care clients responded positively to the first two items, $1 \%$ to the last item, and there was almost no overlap in those identified by the two assessors. We knew that these would be difficult questions to ask, and, in fact, these items were excluded from the test version of the MDS-HC used in Japan because of cultural sensitivity issues.

With respect to the item pool of the MDS-HC, we continue to include a few items with lower Kappa reliability levels. Some, such as fever, are very situation or time dependent, and a consistent answer over a 7-day period is not expected. Other items, although clinically relevant, are diffcult to assess. For example, our work with this instrument and the MDS for nursing homes has indicated that issues related to liquid consumption and dehydration are difficult to evaluate consistently. In addition, there is a limited set of items that were reasonably reliable in one but not the other environment (e.g., change in behavioral symptoms did better in the MDS-HC, whereas weight change did better in the MDS 2.0). For the moment, we assume that these are chance variations, but we do plan to evaluate these items further in subsequent tests.

Finally, as would have been expected, the prevalence of triggered CAP problems increases with increasing cognitive disability. There is a general consistency in this finding, with the severely cognitively impaired group triggering more frequently on bladder, bowel, and risk of institutional placement.

The MDS-HC is now being implemented in agencies in several countries (e.g., Japan, Italy, and the United States), and it now has a variety of support materials, such as a users manual, introductory video, and a data entry and retrieval computer software system. The next steps in our work focus on three areas: developing pre-program screening models, establishing longitudinal benchmarks for expected rates of change in key functional indicators, and developing crossnational models for the distribution of CAPs and the provision of care services. We see these outcome measurements, quality assurance models, and treatment protocols to be natural by-products of the longitudinal data system we are now creating. It is our hope that as a standardized assessment system, the MDS-HC can serve as an internationally valid method of functional assessment. It will serve a role similar to that of the International Classification of Diseases (ICD) in establishing a data base for research and improving the standards of long-term care. ${ }^{26}$

\section{ACKNOWLEDGMENTS}

The authors gratefully acknowledge the contributions of the field trial leaders in the United States, Australia, Canada, the Czech Republic, and Japan, the overall field supervisor at Hebrew Rehabilitation Center for Aged, Research and Training Institute, Pauline Belleville-Taylor, and Yvonne Anderson, secretary. We also acknowledge the cooperation of the home care agencies, field coordinators and MDS-HC assessors, and home care associations (including the National Association for Home Care) who participated in this project. Finally, we acknowledge considerable debt to the inter $R A I$ group and other professionals who assisted in the creation of the RAI-HC system.

\section{REFERENCES}

1. Rubenstein, LZ, Wieland D, Bernabei R. (ieriatric Assessment Technologies: The State of the Art. Milan: Kurris, 1995.

2. Morris JN, Hawes C, Fries BF ct al. Designing the national resident assessment instrument for nursing homes. Gcrontologist 1990;30):293-307.

3. Hawes C, Morris JN, Phillips $(\cdot D)$ et al. Reliability estimates for the minimum data set for nursing home residents and care screening (MDS). Gerontologist 1995;35:172-178.

4. Morris JN, Murphy K, Nonemaker S. I,ong torm care resident assessment instrument user's manual, version 2.0. Washington, DC: American Health Care Association, 1995.

5. Morris JN, Nonemaker S, Murphy K, et al. A commitment to change: Revision of HCYA's RAl. J Am Geriatr Soc 1997;45:1011-1016.

6. Vladek B. From the Health Care Financing Administration. JAMA, $1996 ; 275: 425$.

7. Phillips (I), Morris JN, Hawes C et al. Association of the resident assessment instrument (RAI) with changes in function, cognition, and psychosocial status. J Am Geriatr Soc 1997;45:986-993.

8. Mor $V$, Intrator $O$, Fries $B F$, er al. Changes in hospitalization associated with introducing the RAI. I Am Geriatr Soc 1997;4.5:1002-1010.

9. Challis DJ, Carpenter GI, Traske KJ. Assessment in continuing care homes: Towards a national standard instrument. Canterbury: Joseph Rowntree Foundation/Personal Social Services Research Unit, 1996.

10. Rubenstein 1.Z, Aronow HU, Schloc M, et al. A home-based geriatric assess ment, followup and health promotion program: Design methods and baseline findings from a 3-ycar randomized clinical trial. Aging Clin Exp Res 1994:6:105-120.

11. Rubenstein LZ, Siu AI., Wieland D. Impacts of geriatric evaluation and management programs on defined outcomes. J Am Geriatr Sor 1991;39:8-16.

12. Stuck AF, Siu Al., Wieland GD ct al. Effects of comprehensive geriatric assessment on survival, residence, and function: A metal analysis of controlled trials. Lancet 199.3;342:10.32-10.36.

13. Silverman M, Musa D, Martin DC et al. Evaluation of outpatient geriatric assessment: A randomized mulri-sitc trial, J Am Geriatr Soc 1995;43:733740 .

14. Stuck AE, Aronow HU, Steiner A et al. Trial of annual in-home comprehensive geriatric assessments for elderly people living the community. $N$ Fingl J Med 1995;333:1184-1189.

15. Hirdes, JP, Naus, PJ, Young JK. The use of preventive home visits among frail elderly persons: Evidence from threc European countries. Can J Aging 1994; 13:499-509.

16. Ferrucci L, Guralnik JM, Baroni A et al. Value of combined assessment of physical health and functional status in community-dwelling aged: A prospective study in Florence, Italy. J Gerontol 1991:46:MS2-56.

17. Applegate WB, Blass JP, Williams TF. Instruments for the functional assessment of older patients. N Engl J Med 1990;322:207-1214.

18. Brandeis $(\mathrm{iH}$, Ooi WL, Hossain $\mathrm{M}$ et al. A longitudinal study of risk factors associated with the formation of pressure ulcers in nursing homes. I Am Geriatr Soc 1994:42:388-39.3.

19. Iipsitz I.A, Jonsson PV, Kelley MM, Koestner JS. Causes and correlates of recurrent falls in ambulatory frail elderly. J Gerontol: Med Sci, 1991;46:M114-122.

20. Tinetti ME, Inouye SK, Gill TM, Doucette JT. Shared risk factors for falls, incontinence, and functional dependence: Unifying the approach to geriatric syndromes. JAMA 1995;273:1348-1.354.

21. Calanos AN, Fillenbaum GG, Cohen HJ, Burchett BM. The comprehensive assessment of community dwelling elderly: Why functional status is not enough. Aging Clin Exp Res 1994;6:34,3-3.52. 
22. Steel RK, Sherwood S, Ribbe M. The future: A person-specific standardized assessment instrument. Age Ageing, in press.

23. Fleiss JL. Statistical Methods for Rates and Proportions, 2nd Ed. New York: John Wiley \& Sons, 1981

24. Morris JN, Fries BE, Mehr DR et al. MDS cognitive performance scale. J
Gerontol: Med Sci 1994;49:M174-182.

25. Improving the Quality of care in Nursing Homes. Publication IOM-85-10. Washington DC: National Academy Press, 1986.

26. Ikegami $\mathrm{N}$. Functional assessment and its place in health care. $\mathrm{N}$ Engl J Med 1995;332:598-599. 